\title{
IS LOW ENERGY SUPERGRAVITY CONSISTENT WITH COSMOLOGY AND PARTICLE PHYSICS EXPERIMENTS? *ै
}

\author{
H.E. HABER \\ Department of Physics, University of California, Santa Cruz, CA 95064, USA \\ and \\ G.L. KANE and M. QUIROS ${ }^{1}$ \\ Randall Laboratory of Physics, University of Michigan, Ann Arbor, MI 48109, USA
}

Received 6 June 1985

\begin{abstract}
We construct a low energy supergravity model consistent with all cosmological and particle physics constraints, by omitting some of the usual simplifying assumptions in the hidden sector. The model has heavy squarks, light gluinos and a higgsino as the lightest supersymmetric particle. The experimental implications of the model are discussed. In particular, the possibility of detecting photinos in monojet events, and a new way of producing Higgs bosons in $\mathrm{e}^{+} \mathrm{e}^{-}$collisions with a good signature, $\mathrm{H}^{0}+$ missing energy, occur as new results.
\end{abstract}

Although non-renormalizable, $N=1$ supergravity induces, when spontaneously broken (superHiggs mechanism), an effective (renormalizable) softly broken $N=1$ supersymmetric theory which is valid at energies $\ll \mathrm{O}\left(M_{\mathrm{Pl}}\right)^{\ddagger 1}$ [2]. Moreover, soft breaking terms and/or the top quark Yukawa coupling can trigger electroweak breaking in the low energy effective theory [2] which is, therefore, a good candidate to accommodate the present low energy phenomenology ${ }^{\ddagger 1}[2]$.

Supersymmetric theories normally have a conserved quantum number, $R$-parity, which requires supersymmetric particles to be pair produced and the lightest supersymmetric particle (LSP) to be absolutely stable. This has important consequences: on the one hand, the LSP will contribute to the cosmic density of the universe and might exceed the critical density if it does not annihilate efficiently; on the other hand, at the experimental particle physics level, the cleanest signature for supersymmetry is the missing energy

\footnotetext{
Research supported in part by the US Department of Energy.

1 Permanent address: Instituto de Estructura de la Materia, Serrano 119, 28006 Madrid, Spain.

\$1 For recent reviews see ref. [1].
}

carried away by the LSP, specially at colliders $[3,4]$. In this paper we will search for low energy supergravity models consistent with all cosmological and experimental constraints.

Since missing energy is the basic signature for supersymmetry, the large transverse energy monojets with large missing transverse energy reported by the UA1 Collaboration at the CERN $\mathrm{p} \overline{\mathrm{p}}$ collider [5] are good candidates for an explanation in terms of the production of supersymmetric partners. Different mechanisms were proposed to account for the 1983 UA1 monojets: (a) $\tilde{\text { q }} \overline{\tilde{q}}$ production [6] with subsequent decay $\tilde{\mathrm{q}} \rightarrow \mathrm{q} \tilde{\gamma}$, with $m_{\tilde{\mathrm{q}}}=\mathrm{O}(40) \mathrm{GeV}$ and $m_{\tilde{\mathrm{g}}} \geqslant m_{\tilde{\mathrm{q}}} ;$ (b) $\tilde{\mathrm{g}} \tilde{\mathrm{g}}$ production [7], with decay $\tilde{\mathrm{g}} \rightarrow \mathrm{q} \overline{\mathrm{q}} \tilde{\gamma}, m_{\tilde{\mathrm{g}}}=\mathrm{O}(40) \mathrm{GeV}$ and

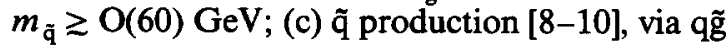
fusion, or $\mathrm{qg} \rightarrow \tilde{\mathrm{q}} \tilde{\mathrm{g}}$ with subsequent decay $\tilde{\mathrm{q}} \rightarrow \mathrm{q} \tilde{\mathrm{g}}$ or $\tilde{\mathrm{q}} \rightarrow \mathrm{q} \tilde{\gamma}, m_{\tilde{\mathrm{q}}} \geq \mathrm{O}(100) \mathrm{GeV}$ and $m_{\tilde{\mathrm{g}}} \simeq \mathrm{O}(3-10)$ $\mathrm{GeV}$.

Recently a general study of all supersymmetric processes giving rise to events similar to those obtained by UA1 has been performed [10]. A reasonable description of the reported data [5] is obtained if monojets come mainly through the decay of massive (100-120 GeV) squarks, with light gluinos, of order $10 \mathrm{GeV}$. The other surviving possibility, $m_{\tilde{\mathrm{q}}} \approx 40 \mathrm{GeV}, m_{\tilde{\mathrm{g}}} \geq 80 \mathrm{GeV}$, is 
difficult to accommodate theoretically [11]. We believe this points toward model (c) above if we wish to have a description consistent with both the reported data and with broken $N=1$ supergravity.

Hereafter we will concentrate on the model with heavy $[\mathrm{O}(100) \mathrm{GeV}]$ squarks and light $[\mathrm{O}(10)$ $\mathrm{GeV}$ ] gluinos. Let us note that the region, in the plane $\left(m_{\tilde{\mathrm{g}}}, m_{\tilde{\mathrm{q}}}\right)$, encompassed by the model is not quite accessible to present beam dump experiments [12], though for gluino masses of $3-5 \mathrm{GeV}$ a slight improvement could detect an effect.

However, the heavy squark and light gluino scenarios have a serious cosmological embarrassment $[9,13]$. The LSP is an (almost) pure photino state which should annihilate into ff by exchanging an $\tilde{f}$. In this way, and on pure dimensional grounds, the annihilation cross section scales as $m_{\tilde{\gamma}}^{2} / m_{\tilde{f}}^{4}$ and the density $\rho_{\tilde{\gamma}}$ as $m_{\tilde{f}}^{4} / m_{\tilde{\gamma}}$. This means that $\rho_{\tilde{\gamma}} \rightarrow \infty$ when $m_{\tilde{\gamma}} \rightarrow 0$ or, in other words, that a lower bound on $m_{\tilde{\gamma}}$, as a function of $m_{\tilde{f}}$, should arise from $\rho_{\tilde{\gamma}}<\rho_{\mathrm{c}}$. This bound has been analyzed by Ellis, Hagelin, Nanopoulos, Olive and Srednicki [14] who found that for $m_{\tilde{\mathrm{f}}} \simeq 100 \mathrm{GeV}$ and $m_{\tilde{\mathrm{g}}} \simeq 10 \mathrm{GeV}, \rho_{\tilde{\gamma}}$ was bigger than $\rho_{\mathrm{c}}$ by two orders of magnitude if we assume the usual relation between photino and gluino masses.

From the cosmological point of view the only way out is to assume that the photino is not the LSP. In that case there are two possible candidates for LSP into which the photino can decay and avoid cosmic superabundance: (a) The sneutrino $\tilde{\nu}_{\tau}[15]$ : this possibility has been ruled out $[11,16]$ in supergravity theories by the UA1 recently reported evidence of the top quark at a mass $m_{\mathrm{t}}=(40 \pm 10) \mathrm{GeV}$ [17] (we disregard the possibility of a fourth generation with a heavy up quark);

(b) The higgsino [14], $\tilde{\chi}$, with a mass $m_{\tilde{\chi}} \leq O(100)$ $\mathrm{eV}$, similar to a massive neutrino, or $m_{\tilde{\chi}} \gtrsim 5 \mathrm{GeV}$, so that $\tilde{\chi} \tilde{\chi}$ could annihilate into $b \bar{b}$.

From now on we will consider the model with heavy squarks, light gluinos and a higgsino with $m_{\tilde{\chi}} \lesssim \mathrm{O}(100) \mathrm{eV}$ or $m_{\tilde{\chi}} \geq 5 \mathrm{GeV}$ as the LSP (the latter possibility, $m_{\tilde{\chi}} \gtrsim 5 \mathrm{GeV}$, is not incompatible with $m_{\tilde{8}} \simeq 10 \mathrm{GeV}$ in our model). Before considering this as a realistic model we have to solve two problems: (i) How is $\mathrm{SU}(2) \times \mathrm{U}(1)$ broken? (ii) Since $\tilde{\chi}$ is lighter than $\tilde{\gamma}$, the decay $\tilde{\gamma} \rightarrow \gamma \tilde{\chi}$ occurs (and is the dominant $\tilde{\gamma}$ decay).
How does this affect the occurrence of monojet events $\$ 2$ ?

As the alert reader may have observed, the $\mathrm{SU}(2) \times \mathrm{U}(1)$ breaking is not accomplished in the usual low energy supergravity way with such a light higgsino. Actually, low energy supergravity $[1,2,19]$ has as free parameters: $m_{3 / 2}$ (gravitino mass), $m_{1 / 2}$ (gaugino mass at $M_{\mathrm{GUT}}$ ), $A$ [dimensionless parameter of $O(1)]$ and $m_{4}$ (a supersymmetric higgsino mass).

The soft breaking parameters $m_{3 / 2}$ and $A$ are related to the super-Higgs mechanism: it is usually assumed the total superpotential $f_{\text {TOT }}=M^{2} f_{2}(\zeta)$ $+g\left(y^{a}\right)$, where $\zeta \equiv z / M$ is the field responsible for supersymmetry breaking and $y^{a}$ are the observable fields. In that case $m_{3 / 2} \propto\left|f_{2}(\langle\zeta\rangle)\right|$ and $A=\sqrt{3}\langle\zeta\rangle$ [19].

For a light top quark, i.e., $m_{\mathrm{t}} \simeq 40 \mathrm{GeV}$, the vacuum expectation values of Higgs doublets coupled to up- and down-type quarks are similar, $\left\langle\mathrm{H}_{1}\right\rangle \simeq\left\langle\mathrm{H}_{2}\right\rangle$, and the neutralino four-by-four mass matrix has a pure higgsino mass eigenstate $\tilde{\chi}$ with a mass $m_{\tilde{\chi}} \simeq m_{4}[20]$. On the other hand, for such a light top quark, the parameter which governs the electroweak radiative breaking, $m_{3}^{2}$, is related to $m_{4}$ by $m_{3}^{2}=(A-1) m_{3 / 2} m_{4}$ (at $\left.M_{\mathrm{GUT}}\right)$. But in that case the condition $m_{3}=$ $\mathrm{O}\left(M_{\mathrm{W}}\right)$, necessary for $\mathrm{SU}(2) \times \mathrm{U}(1)$ breaking, is incompatible with the condition $m_{4} \ll M_{\mathrm{W}}$ necessary for the higgsino to be the LSP.

The essential problem here is that the same parameter $m_{4}$ governs the higgsino mass and the electroweak breaking. The way out is to construct a model where $m_{4}$ and $m_{3}^{2}$ get decoupled. To do that we have used the general method introduced by Soni and Weldon [21] to extract the most general low energy theory from $N=1$ supergravity. In particular if one introduces the total superpotential

$f_{\text {TOT }}=M^{2} f_{2}(\zeta)+g\left(y^{a}\right)+\omega(\zeta) h\left(y^{a}\right)$,

where $\omega(\langle\zeta\rangle)=0$, the low energy theory has one more parameter, related to $\omega^{\prime}(\langle\zeta\rangle)$. This translates into the fact that $m_{3}^{2}$ and $m_{4}$ get decoupled as

\#2 Searches for $\tilde{\gamma} \rightarrow \gamma+\tilde{\chi}$, where $\tilde{\chi}$ escapes the detector, at $\mathrm{e}^{+} \mathrm{e}^{-}$colliders are not inconsistent with our model since our selectron mass is about $110 \mathrm{GeV}$, while the data is only sensitive to $\tilde{m}_{\ell}<100 \mathrm{GeV}$ [18]. 
boundary conditions, and permits $\mathrm{SU}(2) \times \mathrm{U}(1)$ breaking with a higgsino mass $m_{\bar{x}} \ll M_{\mathrm{w}}$. It should be emphasized that the Soni-Weldon method gives the most general low energy theory and that the term involving $\omega$ was arbitrarily left out in the past. The details of the more general low energy theory as well as the electroweak radiative breaking will be found in ref. [11].

As for the second problem, it is clear that $\tilde{\gamma}$ will decay into $\gamma \tilde{\chi}$ and the decay rate will depend on $m_{\tilde{\gamma}}^{2}-m_{\tilde{\chi}}^{2}$. We have studied (see below) the decay rate $\Gamma(\tilde{\gamma} \rightarrow \gamma \tilde{\chi})$ and concluded that if $m_{\tilde{\gamma}}=\frac{8}{3} \times$ $\sin ^{2} \theta_{\mathrm{w}}\left(\alpha_{2} / \alpha_{3}\right) m_{\overline{\mathrm{g}}}$, as in the usual models when all gaugino Majorana masses are equal at $M_{\mathrm{GUT}}$, then for $m_{\tilde{\mathrm{g}}} \simeq 10 \mathrm{GeV}$ all photinos should decay inside the UA1 detector and all monojets should be accompanied by a photon. While this may not be excluded by the data (since photons look like $\pi^{0}$ 's), it stimulated us to examine the origin of gaugino Majorana masses in the low energy theory.

In $N=1$ supergravity, gaugino Majorana masses appear only from non-canonical kinetic terms in the gauge sector. Actually [2] $m_{\alpha \beta} \propto$ $\partial f_{\alpha \beta / \partial \phi^{i}}$ where $\phi^{i}$ are all the fields of the theory, so that if one assumes $f_{\alpha \beta}=\delta_{\alpha \beta}$ then gaugino Majorana masses can never be generated. One usually implicitly assumes the simplest ansatz $f_{\alpha \beta}=A(z) \delta_{\alpha \beta}$ which leads to the equality of all gaugino masses at $M_{\mathrm{GUT}}, M_{1}=M_{2}=M_{3}$, and thus to the above relation between $m_{\tilde{g}}$ and $m_{\tilde{\gamma}}$. On the other hand, it is clear that there is no symmetry argument behind such an ansatz (simplicity is not a symmetry) because $\left\langle f_{\alpha \beta}\right\rangle$ has only to be $\mathrm{SU}(3) \times \mathrm{SU}(2) \times \mathrm{U}(1)$ invariant. The more general ansatz [22]

$f_{\alpha \beta}=A(z) \delta_{\alpha \beta}+B(z) d_{\alpha \beta \gamma} \Sigma^{\gamma}$,

where $\Sigma^{\gamma}$ is in the 24 representation of SU(5), is equally acceptable, and provides one more free parameter in the low energy theory, related to $B^{\prime}(\langle z\rangle)$. If we choose $B(\langle z\rangle)=0$ then $\alpha_{1}=$ $\alpha_{2}=\alpha_{3}$ at $M_{\text {GUT }}$ and the GUT predictions $\left(M_{\mathrm{GUT}}, \sin ^{2} \theta_{\mathrm{w}}, m_{\mathrm{b}} / m_{\tau}\right)$ are unchanged. We can now introduce a real parameter $\lambda$ and write

$m_{\tilde{\gamma}}=\lambda \frac{8}{3} \sin ^{2} \theta_{\mathrm{w}}\left(\alpha_{2} / \alpha_{3}\right) m_{\tilde{g}}$.

In that case the three gaugino Majorana masses at $M_{\mathrm{GUT}}, M_{1}, M_{2}, M_{3}$, scale as [11]

$M_{3} / 15=M_{2} /(20 \lambda-5)=M_{1} /(12 \lambda+3)$.

Although we have assumed an SU(5) GUT, the procedure can be worked out in any GUT and the result is that $m_{\tilde{\gamma}}$ and $m_{\tilde{\mathrm{g}}}$ can be considered as independent degrees of freedom. In our particular model we will use this additional freedom to fix $m_{\tilde{\gamma}}$ from the decay rate $\Gamma(\tilde{\gamma} \rightarrow \gamma \tilde{\chi})$ when it is measured (and constrain it for now).

To summarize the situation, our model in its more general form has six parameters: $m_{3 / 2}$, $m_{1 / 2}, m_{3}^{2}, m_{4}, A$ and $m_{\tilde{\gamma}} . m_{3 / 2}$ and $m_{1 / 2}$ are fixed by $m_{\tilde{\mathrm{q}}}$ and $m_{\tilde{\mathrm{g}}}, m_{4}^{2}$ by $m_{\tilde{x}}, m_{3}$ by the condition for $\mathrm{SU}(2) \times \mathrm{U}(1)$ breaking, and $m_{\tilde{\gamma}}$ (or better $\left.m_{\tilde{\gamma}}-m_{\tilde{\chi}}\right)$ by the decay rate $\Gamma(\tilde{\gamma} \rightarrow \gamma \tilde{\chi})$. Only $A$ remains as a free parameter and it has to respect the stability bound of the potential and avoid $\mathrm{SU}(3)_{\mathrm{c}} \times \mathrm{U}(1)_{\mathrm{em}}$ breaking minima.

The supersymmetric spectrum is almost determined once we fix $m_{\tilde{\mathrm{q}}}$ and $m_{\tilde{\mathrm{g}}}$. In the limit $v_{2} / v_{1} \rightarrow 1$ (which corresponds to small top quark mass, i.e., $m_{\mathrm{t}} \simeq 40 \mathrm{GeV}$ ) and neglecting the renormalization due to gaugino masses, which is less than $1 \%$ in our model, we obtain the following spectrum:

(a) All sleptons and five flavors of squarks are essentially degenerate in mass: $m_{\tilde{\ell}}=m_{\tilde{\mathrm{q}}}=m_{3 / 2}$. Only the top squark is split by the $A$ parameter which contributes to the non-diagonal mass term $\tilde{\mathrm{t}}_{\mathrm{L}}^{*} \tilde{\mathrm{t}}_{\mathrm{R}}+\tilde{\mathrm{t}}_{\mathrm{R}}^{*} \tilde{\mathrm{t}}_{\mathrm{L}}$. The mass eigenstates have a mass: $m_{\tilde{t}_{1,2}}^{2}=m_{\tilde{\mathrm{q}}}^{2}+m_{\mathrm{t}}^{2} \pm A m_{\mathrm{t}} m_{\tilde{\mathrm{q}}}$.

(b) Three neutral higgses with masses: $M_{\mathrm{H}_{1}}^{2}=$ $2 m_{\tilde{\mathrm{q}}}^{2}, m_{\mathrm{H}_{2}}^{2}=m_{\mathrm{H}_{1}}^{2}+M_{\mathrm{Z}}^{2}$, and the third one which gets a mass from radiative corrections [20]

$$
\begin{aligned}
& m_{\mathrm{rad}}^{2}=\left\{\left(3 \alpha_{2} / 4 \pi\right)\left[1+\left(A^{2}+2\right)\left(m_{\mathrm{t}} / M_{\mathrm{W}}\right)^{2}\right]\right. \\
& \left.\quad+3 \alpha_{1} / 20 \pi\right\} m_{\tilde{\mathrm{q}}}^{2} .
\end{aligned}
$$

(c) Two charged higgses with a mass: $m_{\mathrm{H}_{ \pm}}^{2}=$ $2 m_{\tilde{\mathrm{q}}}^{2}+M_{\mathrm{W}}^{2}$.

(d) The charged winos combine with charged higgsinos and form a pair of Dirac particles with a mass $M_{\mathrm{W}}$.

(e) The neutralinos are, apart from the higgsino $\tilde{\chi}$ and photino $\tilde{\gamma}$ whose masses are input, a pair of Majorana spinors degenerate in mass, $M_{\tilde{z}}=M_{z}$, which combine into a Dirac spinor. 
The above formulas are only approximate since $v_{2} \neq v_{1}$. Actually for $m_{3 / 2}=110 \mathrm{GeV}$ and $m_{\tilde{\mathrm{g}}}=$ $12 \mathrm{GeV}$ we find [11] $v_{2} / v_{1}=1.03,1.05,1.08$, and 1.10 for $A=0,1,2$ and 2.4. When the corrections in $1-\left(v_{2} / v_{1}\right)^{2}$ are considered there is a small splitting between left and right sfermions but the largest splitting occurs in the stop sector due to the $A$-parameter. We find that the condition [23] $m_{\mathrm{t}} \leqslant m_{\tilde{\mathrm{i}}}+m_{\tilde{\gamma}}$, which seems to be necessary to avoid the decay $\mathrm{t} \rightarrow \tilde{\mathrm{t}} \tilde{\gamma}$, corresponds to the bound $|A| \leq 2.4$. The lightest neutral Higgs is the radiative one, with a mass: $m_{\mathrm{rad}}=12 \mathrm{GeV}$, $13 \mathrm{GeV}, 15.5 \mathrm{GeV}$ and $16.2 \mathrm{GeV}$, for the above values of $\boldsymbol{A}$ (neglecting tree level contributions); this Higgs is too heavy to be produced in $\Upsilon$ decays. Also the neutralino $\tilde{Z}$ splits into two Majorana fermions with masses $M_{\mathrm{Z}} \pm \Delta$ with $\Delta<1 \mathrm{GeV}$. This has phenomenological consequences as we will see later. For more details on the supersymmetric spectrum see ref. [11].

Theoretical and experimental constraints have left free the parameter $A$, with $|A| \leqslant 2.4$, and the photino and higgsino masses. These are constrained as we will see to $m_{\tilde{\gamma}}-m_{\tilde{\chi}}<$ few hundred $\mathrm{MeV}$ if the photino lives long enough to escape the UA1 detector. Corresponding to the two cosmologically allowed possibilities, $m_{\tilde{\chi}} \leqslant 100 \mathrm{eV}$ or $m_{\tilde{\chi}} \geq m_{\mathrm{b}}$, we conclude that either $m_{\tilde{\gamma}} \leqslant$ few hundred $\mathrm{MeV}$ or $m_{\tilde{\gamma}} \simeq 5 \mathrm{GeV}$. If the analysis of the monojet data turns out to imply $m_{\tilde{g}} \leqslant m_{\mathrm{b}}$ it could happen that only the lighter photino alternative would survive. We assume a massless higgsino in the following, unless explicitly stated otherwise. Let us consider a number of phenomenological implications:

(a) Since $m_{\tilde{\chi}}<m_{\tilde{\gamma}}$, the dominant decay of $\tilde{\gamma}$, $\tilde{\gamma} \rightarrow \gamma \tilde{\chi}$, occurs at one loop (it is a triangle diagram

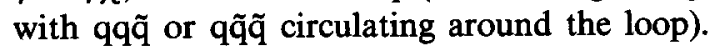
While we were computing this decay the paper of ref. [24] arrived. Except for a color factor of 9 omitted in ref. [24] which decreases the lifetime an order of magnitude we agree with their results. In addition [11] there are diagrams with $\mathrm{W}^{+} \tilde{\chi}^{-} \tilde{\chi}^{-}$, $\mathbf{W}^{+} \mathbf{W}^{+} \tilde{\chi}^{-}, \mathbf{H}^{+} \tilde{\chi}^{-} \tilde{\chi}^{-}$and $\mathbf{H}^{+} \mathbf{H}^{+} \tilde{\chi}^{-}$circulating around the loop ( $\tilde{\chi}^{-}$is a chargino), but their contribution is suppressed. Neglecting the $\tilde{\mathrm{t}}_{\mathrm{L}}-\tilde{\mathrm{t}}_{\mathrm{R}}$ mixing, which corresponds to the case $A=0$, we obtain for the photino width [11] from just the quark-squark loops,

$$
\begin{aligned}
\Gamma= & \left(9 G_{\mathrm{F}} e_{\mathrm{t}}^{4} \alpha^{2} \cot ^{2} \theta / 4 \sqrt{2} \pi^{3}\right) m_{\tilde{\gamma}}^{3} \\
& \times\{[1 /(1-r)](1+[r /(1-r)] \log r)\}^{2},
\end{aligned}
$$

where $e_{\mathrm{t}}$ is the electric charge of the top quark, $\cot \theta=v_{1} / v_{2}$ and $r=\left(m_{\tilde{t}} / m_{\mathrm{t}}\right)^{2}$. Including mixing there is a correction of order $m_{\tilde{\gamma}} / m_{\mathrm{t}}$ which is numerically small. For our model $r=8.7$ so that $\Gamma=2.2 \times 10^{-13}\left[m_{\bar{\gamma}}(\mathrm{GeV})\right]^{3} \mathrm{GeV}$ and a photino of energy $40 \mathrm{GeV}$ would travel $\gamma c \tau \simeq 0.04 /\left[m_{\tilde{\gamma}}\right.$ $(\mathrm{GeV})]^{4} \mathrm{~m}$. Therefore for $m_{\bar{\gamma}} \leq 0.25 \mathrm{GeV}$ all photinos would escape a collider detector while for $m_{\tilde{\gamma}} \geq 0.5 \mathrm{GeV}$ all photinos would decay within the detector. Since the decay $\tilde{\gamma} \rightarrow \gamma \tilde{\chi}$ gives a flat energy spectrum from 0 to $E_{\tilde{\gamma}}$ for the $\tilde{\chi}$, some expected monojet events would have an energetic $\tilde{\chi}$ and remain monojet events, while others would have $E_{\tilde{\chi}} \ll E_{\tilde{\gamma}}$ and not pass the UA1 cuts as monojet events. Events with isolated hard photons would occur, but not with large accompanying missing momentum. Depending on the photino lifetime, it could happen that a large number of photinos would escape the detector before decaying, giving rise to isolated photons which suddenly appear some meters from the collider detector. They could be searched for by surrounding part of the large angle region of the detector at a distance of some meters with a photon detector! The photons would point back to the interaction approximately, and occur in timing coincidence with the monojet event. If such a situation occurred essentially every photino could be detected, instead of having to wait decade(s) as with neutrinos to find explicitly the carrier of missing momentum.

Observation of the photons would not only confirm the entire viewpoint considered here, it would also allow determination of $m_{\tilde{g}}$ and $m_{\tilde{\gamma}}$. More details of the calculation of $\Gamma(\tilde{\gamma} \rightarrow \gamma \tilde{\chi})$, including the case of $\tilde{\mathrm{t}}_{\mathrm{L}}-\tilde{\mathrm{t}}_{\mathrm{R}}$ mixing, i.e., $A \neq 0$, can be found in ref. [11].

(b) In a large class of supersymmetric theories with light higgsinos, a new opportunity to discover a Higgs boson presents itself. Rather generally, there is a mass $M_{\tilde{z}_{1}}<M_{\mathrm{z}}$. Then the decay $\mathrm{Z} \rightarrow \tilde{\chi} \tilde{Z}$. occurs at tree level with a strength of 
order the usual $\mathrm{Z} \rightarrow \mathrm{f} \overline{\mathrm{f}}$ modes (such as $\mathrm{Z} \rightarrow \mu^{+} \mu^{-}$) suppressed only by phase space. Once that decay has occurred, the $\tilde{Z}_{1}$ can decay via $\tilde{Z}_{1} \rightarrow \tilde{\chi} \mathrm{H}^{0}$ or $\tilde{Z}_{1} \rightarrow \mathrm{q} \overline{\mathrm{q}} \tilde{\mathrm{g}}$ or $\tilde{\mathrm{Z}}_{1} \rightarrow \tilde{\chi} \mathrm{f} \overline{\mathrm{f}}$. These turn out to be comparable in size and the $\tilde{\chi} \mathrm{H}^{0}$ mode can dominate, essentially independently of $m_{\mathrm{H}^{0}}$, if $m_{\mathrm{H}^{0}} \leqslant 60-70 \mathrm{GeV}$. Thus it can easily happen that $\mathrm{e}^{+} \mathrm{e}^{-} \rightarrow \mathrm{Z}^{0} \rightarrow \tilde{\chi} \tilde{\chi} \mathrm{H}^{0}$ occurs at the level of $10^{-3}$ or more of all $\mathbf{Z}^{0}$ decays. This gives a very clear signature, with a one-sided event from the $\mathrm{H}^{0}$ decay and the two escaping $\tilde{\chi}$. In our model $12 \mathrm{GeV} \leqslant m_{\mathrm{H}^{0}} \leqslant 16 \mathrm{GeV}$, so $\mathrm{H}^{0} \rightarrow b \bar{b}$. If monojets are real and related to supersymmetry this is a highly likely situation, and could be the most probable way to detect a Higgs boson. Although this mechanism is suppressed for the particular spectrum we have given as an example, with $m_{\tilde{\mathrm{Z}}} \simeq m_{\mathrm{Z}}$ and $v_{1} \simeq v_{2}$, it could still be of great importance for different parameters. More details of these calculations will be presented elsewhere [25]:

(c) Several interesting $\mathrm{Z}^{0}$ decays may or may not occur:

(i) If $\mathrm{t}$ is light enough the $\mathrm{Z} \rightarrow \mathrm{t} \mathrm{t} p_{\mathrm{T}}$, giving some extra events which could appear to come from a heavier $\mathrm{Z}$ if the missing momentum were assigned to a single $\nu$.

(ii) $\mathrm{Z} \rightarrow \tilde{\mathrm{g}} \tilde{\mathrm{g}}$ has a branching ratio which varies as $A$ varies from 0 to 2.4 [26], so this small mode can eventually be detected and $A$ can be determined.

(iii) The effect on $\Gamma_{z}$ is small, as no squarks or slepton modes are energetically allowed, $Z \rightarrow \tilde{\chi} \tilde{\chi}$ is suppressed by a factor $\left(v_{2}-v_{1}\right)^{2} / v^{2}$, and so the largest new mode is $\tilde{\chi} \tilde{Z}_{1}$.

(d) A $\widetilde{\mathrm{tt}}$ pair can be directly produced and detected at a hadron collider. The cross section is about $1 / 4$ that for a quark of the same mass, but the signature could be better because of the $\tilde{\gamma}$ or $\tilde{\chi}$ in the decay. Measuring $m_{\mathfrak{t}}$ from the event structure, or the production cross section, determines the parameter $A$.

(e) There should be no direct production of any sleptons or squarks at SLC, LEP, HERA or TRISTAN in our model.

(f) The gluino has a branching ratio $\tilde{\mathrm{g}} \rightarrow \mathrm{g} \tilde{\gamma}$ which can be as large as $3 \%$ in our model [27]. The branching ratio determines $m_{\mathfrak{i}}$ and $A$ if it is measured, which could be possible when several hundred monojet events are available.

(g) In the photon counting experiments $\mathrm{e}^{+} \mathrm{e}^{-} \rightarrow$ $\gamma+\not \mathbb{t}$ there will be no signal from the usual diagrams since $m_{\ell} \geq 100 \mathrm{GeV}$, but some small contribution can occur from the higgsino final state if $v_{1} \neq v_{2}$.

(h) As far as we are aware, no interesting flavor changing neutral current effects are induced.

(i) Since $\tilde{\chi}$ is the LSP, if $m_{\tilde{\chi}} \leq 100 \mathrm{eV}$, $\tilde{\chi}$ is a good candidate for hot dark matter, and for contributing to stellar energy loss. If $m_{\tilde{x}} \simeq m_{\mathbf{b}}$, then $\tilde{\chi}$ may be a good candidate for cold dark matter.

In conclusion we have constructed a low energy supergravity model consistent with all experimental constraints (including the cosmological ones). The model has heavy squarks $(\sim 100 \mathrm{GeV})$, light gluinos $(\sim 10 \mathrm{GeV})$ and the higgsino as the lightest supersymmetric particle. In order to break $S U(2) \times U(1)$ the higgsino mass is decoupled from the scale of electroweak breaking by omitting some of the usual simplifying assumptions in the hidden sector of $N=1$ supergravity, but still with canonical kinetic terms (i.e., minimal coupling) in the chiral sector. The photino mass is decoupled from the gluino mass by means of a general non-minimal coupling in the gauge sector. We discuss the experimental implications of the model. One interesting consequence is the possibility of detecting the photinos that provide the missing momentum in monojet events by surrounding part of the large angle region of the detector at some distance with a photon detector. Depending on the gluino lifetime, it may be important to design beam dump experiments to detect the photons from photino $\rightarrow$ photon + higgsino (the photinos having come from gluino decay). Another consequence of the model is a new way of producing Higgs bosons in $\mathrm{e}^{+} \mathrm{e}^{-} \rightarrow \mathrm{Z}^{0}$ $\rightarrow \tilde{\chi} \tilde{Z} \rightarrow \tilde{\chi} \tilde{\chi} \mathbf{H}^{0}$, giving a signature, $\mathrm{H}^{0}+$ missing energy, which may be good for detection.

\section{References}

[1] J. Ellis, CERN preprint TH-3802 (1984); D.V. Nanopoulos, CERN preprint TH-3995 (1984); 
P. Nath, R. Arnowitt and A.H. Chamseddine, Applied $N=1$ Supergravity, ICTP Series in Theoretical Physics, Vol. I (World Scientific, Singapore, 1985).

[2] H.P. Nilles, Phys. Reg. 110 (1984) 1.

[3] G.L. Kane and J.P. Leveille, Phys. Lett. 112B (1982) 227

[4] H.E. Haber and G.L. Kane, Phys. Rep. 117 (1985) 75.

[5] UA1 Collab., G. Arnison et.al., Phys. Lett. 139B (1984) 115;

M. Mohammedi, invited talk 1st Annual Aspen Winter Physics Conf. (Aspen, Colorado, January 1985).

[6] J. Ellis and H. Kowalski, Phys. Lett. 142B (1984) 441; 157B (1985) 416 Nucl. Phys. B246 (1984) 189; preprint CERN-TH-4072 (1984);

A.R. Allan, E.W.N. Glover and A.D. Martin, Phys. Lett. 146B (1984) 247;

A.R. Allen, E.W.N. Glover and S.L. Grayson, Durham preprint DTP/84/28 (1984);

V. Barger, K. Hagiwara and W.-Y. Keung, Phys. Lett. 145B (1984) 147; Wisconsin preprint MAD/PH/197 (1984).

[7] E. Reya and D.P. Roy, Phys. Rev. Lett. 51 (1983) 867; 51 (1983) 1307(E); 53 (1984) 881; Phys. Lett. 141 B (1984) 442; Dortmund preprint DO-TH 84-19 (1984); M. Glück, E. Reya and D.P. Roy, Phys. Lett. 155B (1985) 284.

[8] M.J. Herrero, L.E. Ibán̄ez, C. López and F.J. Yndurain, Phys. Lett. 132B (1983) 199; 145B (1984) 430; V. Barger, K. Hagiwara, W. Keung and J. Woodside, Phys. Rev. Lett. 53 (1984) 641; Madison preprint MAD/PH/197 (1984).

[9] V. Barger, S. Jacobs, J. Woodside and K. Hagiwara, Madison preprint MAD/PH/232 (1985);

A. De Rújula and R. Petronzio, CERN preprint TH-4070 (1984).

[10] R.M. Barnett, H.E. Haber and G.L. Kane, Phys, Rev. Lett. 54 (1985) 1983.

[11] M. Quirós, G.L. Kane and H.E. Haber, University of Michigan preprint UM-TH-85-10 (1985).

[12] F. Bergsma et al., Phys. Lett. 121B (1983) 429;
R.C. Ball et al., Phys. Rev. Lett. 53 (1984) 1314.

[13] J. Ellis, Talk p $\bar{p}$ collider meeting (St. Vincent, February 1985);

S. Nandi, University of Texas preprint (1985).

[14] J. Ellis, J.S. Hagelin, D.V. Nanopoulos, K. Olive and M. Srednicki, Nucl. Phys. B238 (1984) 453.

[15] J.S. Hagelin, G.L. Kane and S. Raby, Nucl. Phys. B241 (1984) 638;

L.E. Iban̄ez, Phys. Lett. 137B (1984) 160.

[16] B. Gato, J. León, J. Pérez-Mercader and M. Quirós, preprint IEM-HE-5 (1985), Nucl. Phys. B, to be published.

[17] UA1 Collab., G. Arnison et.al., Phys. Lett. 147B (1984) 493.

[18] E. Ros, 13th Intern. Winter Meeting on Fundamental Physics (Cuenca, Spain, April 1985).

[19] R. Barbieri, S. Ferrara and C.A. Savoy, Phys. Lett. 119B (1982) 343;

L. Hall, J. Lykken and S. Weinberg, Phys. Rev. D27 (1983) 346;

H.P. Nilles, M. Srednicki and D. Wyler, Phys. Lett. 120B (1983) 346.

[20] C. Kounnas, A.B. Lahanas, D.V. Nanopoulos and M. Quirós, Nucl. Phys. B236 (1984) 438; Phys. Lett. 132B (1983) 95.

[21] S.K. Soni and H.A. Weldon, Phys. Lett. 126B (1983) 215.

[22] J. Ellis, C. Kounnas and D.V. Nanopoulos, Nucl. Phys. B247 (1984) 373;

J. Ellis, K. Enqvist, D.V. Nanopoulos and K. Tamvakis, CERN preprint TH-4018 (1985).

[23] G.L. Kane, Lectures 1984 SLAC Summer Institute on Heavy quarks;

J. Ellis, CERN preprint TH-4017 (1984).

[24] H. Komatsu and J. Kubo, Phys. Lett. 157B (1985) 90.

[25] H.E. Haber, G.L. Kane, I. Kani and M. Quirós, University of Michigan preprint (in preparation).

[26] G.L. Kane and W.B. Rolnick, Nucl, Phys. B217 (1983) 117 ; W.B. Rolnick (in preparation).

[27] H.E. Haber and G.L. Kane, Nucl. Phys. B232 (1984) 333. 